\title{
Systematic approach to selecting licensed drugs for repurposing in the treatment of progressive multiple sclerosis
}

\author{
Nick Cunniffe (D) , ${ }^{1}$ Khue Anh Vuong, ${ }^{2}$ Debbie Ainslie, ${ }^{3}$ David Baker (D) , ${ }^{4}$ \\ Judy Beveridge, ${ }^{3}$ Sorrel Bickley, ${ }^{2}$ Patrick Camilleri, ${ }^{5}$ Matthew Craner, $^{6}$ \\ Denise Fitzgerald, ${ }^{7}$ Alerie $G$ de la Fuente, ${ }^{7}$ Gavin Giovannoni (i) , ${ }^{4}$ Emma Gray, ${ }^{2}$ \\ Lorraine Hazlehurst, ${ }^{3}$ Raj Kapoor, ${ }^{8}$ Ranjit Kaur, ${ }^{3}$ David Kozlowski, ${ }^{3}$ Brooke Lumicisi, ${ }^{2}$ \\ Don Mahad ${ }_{1}^{9}$ Björn Neumann, ${ }_{1}^{1}$ Alan Palmer, ${ }^{10}$ Luca Peruzzotti-Jametti, ${ }^{1}$ \\ Stefano Pluchino, ${ }^{1}$ Jennifer Robertson, ${ }^{2}$ Alan Rothaul, ${ }^{11}$ Lyndsey Shellard, ${ }^{3}$ \\ Kenneth J Smith, ${ }^{12}$ Alastair Wilkins, ${ }^{13}$ Anna Williams, ${ }^{14}$ Alasdair Coles ${ }^{1}$
}

For numbered affiliations see end of article.

Correspondence to Dr Nick Cunniffe, Department of Clinical Neurosciences, University of Cambridge, Cambridge CB2 OQQ, UK; ngc26@cam.ac.uk

Received 16 June 2020 Revised 8 September 2020 Accepted 13 October 2020 Published Online First 12 November 2020

\section{Check for updates}

(C) Author(s) (or their employer(s)) 2021. No commercial re-use. See rights and permissions. Published by BMJ.

To cite: Cunniffe N, Vuong KA, Ainslie D, et al. J Neurol Neurosurg Psychiatry

2021:92:295-302

\section{ABSTRACT}

Objective To establish a rigorous, expert-led, evidencebased approach to the evaluation of licensed drugs for repurposing and testing in clinical trials of people with progressive multiple sclerosis (MS).

Methods We long-listed licensed drugs with evidence of human safety, blood-brain barrier penetrance and demonstrable efficacy in at least one animal model, or mechanistic target, agreed by a panel of experts and people with MS to be relevant to the pathogenesis of progression. We systematically reviewed the preclinical and clinical literature for each compound, condensed this into a database of summary documents and shortlisted drugs by scoring each one of them. Drugs were evaluated for immediate use in a clinical trial, and our selection was scrutinised by a final independent expert review.

Results From a short list of 55 treatments, we recommended four treatments for immediate testing in progressive MS: R- $\alpha$-lipoic acid, metformin, the combination treatment of R- $\alpha$-lipoic acid and metformin, and niacin. We also prioritised clemastine, lamotrigine, oxcarbazepine, nimodipine and flunarizine.

Conclusions We report a standardised approach for the identification of candidate drugs for repurposing in the treatment of progressive MS.

\section{INTRODUCTION}

Multiple sclerosis (MS) is a chronic, primarily inflammatory, disorder of the central nervous system in which demyelination occurs alongside axonal and neuronal degeneration. ${ }^{1}$ There now exists an extensive therapeutic armamentarium for the $85 \%$ of patients presenting with episodic neurological dysfunction (relapsing remitting MS (RRMS)). ${ }^{2}$ However, the expanding repertoire of these anti-inflammatory disease-modifying treatments contrasts with a paucity of effective therapies for the $15 \%$ of people that present with progressive disability (primary progressive MS) and indeed the $80 \%$ of patients with RRMS who subsequently develop progression (secondary progressive MS
(SPMS)). ${ }^{3}$ While ocrelizumab and siponimod have shown modest benefits in phase III trials, ${ }^{45}$ most immunotherapies have failed in non-active progressive disease. Finding drugs to treat progression remains the greatest unmet need for people with MS.

The reasons for the lack of an effective therapy for progressive MS are multifaceted. The pathophysiology of progressive MS is poorly understood (reviewed in ref 6), and there is no animal model that accurately mimics the entirety of the disease. So, new target and drug discovery are challenging. Drug repurposing is attractive, with fewer hurdles before reaching clinical trials, but the rationale behind drug selection needs to be carefully considered. $^{78}$

In 2011, the MS Society sponsored an initiative to choose licensed drugs to be trialled in secondary progressive MS. ${ }^{9}$ Only oral treatments with a putative action against neurodegeneration were considered. Highest priority was given to drugs that had been tested in MS, Alzheimer's disease, motor neuron disease/amyotrophic lateral sclerosis, Parkinson's disease and/or Huntington's disease. Clinical and laboratory data from each drug were brought, in a standard template, to a panel composed of people with MS and experts in animal models, disease biology, clinical trial design and systematic review. The final panel treatment selection was: riluzole, amiloride, fluoxetine, ibudilast, oxcarbazepine, pirfenidone and agents of the polyunsaturated fatty acid (PUFA) class (including lipoic acid). Of these, both ibudilast and lipoic acid have since shown efficacy in progressive MS in phase II trials. ${ }^{10} 11$ The Multiple Sclerosis Secondary Progressive Multi-Arm Randomisation Trial study tested riluzole, amiloride and fluoxetine versus placebo in 445 people with SPMS. ${ }^{12}$ Unfortunately, no treatment effect on brain atrophy (percentage brain volume change) was seen over 2 years. ${ }^{13}$

In 2018, the MS Society set up an expert consortium (figure 1) to select treatments and design a new phase of drug trials in progressive MS utilising a novel 

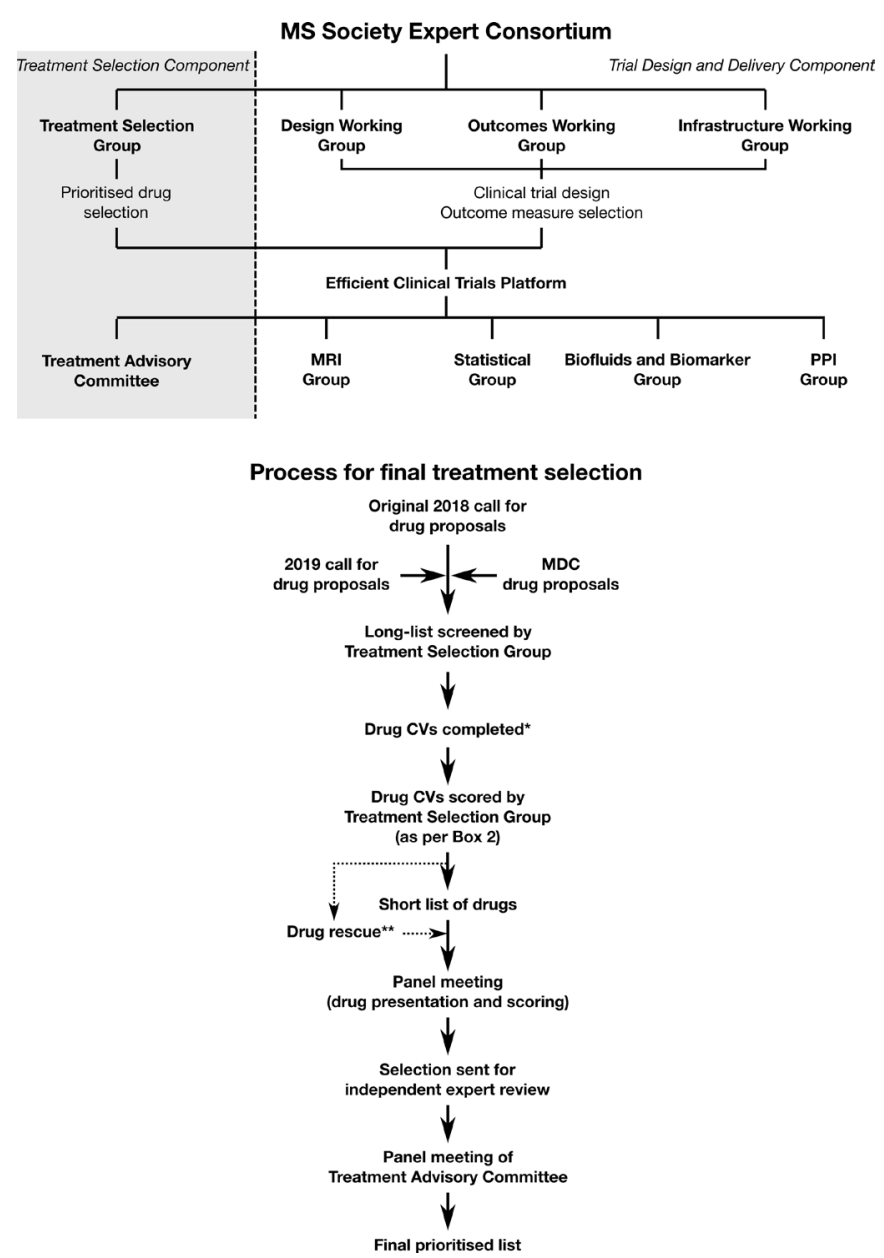

Figure 1 Above: summary of the UK MS Society's expert consortium for progressive MS clinical trials, which has been set up to test treatments in an adaptive platform trial, termed the efficient clinical trials platform. The treatment selection group and treatment advisory committee were responsible for formulating the prioritised list of drugs to enter the clinical trial. Below: flow chart of the procedures undertaken during the final round of treatment selection by the treatment selection element. *Drug Curriculum Vitaes (CVs) were completed by four members of the treatment selection group - two with a scientific background and two MS specialist clinicians. * ${ }^{*}$ Drugs failing to reach the short list of drugs on account of a low score could be added back for consideration at the panel meeting if reasons were proposed by a member of the treatment selection group and its rescue approved by majority vote. MDC, medicines discovery catapult; MS, multiple sclerosis; PPI, patient and public involvement.

adaptive methodology. Working as the treatment selection component of this consortium, we augmented the previous strategy with an expert and mechanism-led approach, which we describe here.

\section{METHODS}

\section{Pilot stage of treatment selection}

The original treatment selection group included 10 scientific members (specialist MS clinicians, laboratory scientists and people with experience of the pharmaceutical industry) and two people with MS. The latter were selected from the MS Society's Research Network $(\mathrm{RN})$ : a group of people living with, or caring for, someone with MS, who are trained and experienced in working with researchers to strengthen the quality and relevance of research by drawing on their personal experience of MS.
At the first meeting of the treatment selection group in January 2018, the following principles of treatment selection were agreed: the highest priority would be given to safe licensed drugs acting on pathological mechanisms thought to be relevant to progression in MS, including remyelination; to drugs that cross the blood-brain barrier; and those that had demonstrable efficacy in at least one relevant animal model. Experience of the drug's use in MS or any other neurological illnesses was considered but did not weight treatment choice. Immunotherapies, such as B cell depleting drugs, were excluded, given the considerable industrial investment in this area. The agreed mechanistic areas were: (1) energy, blood flow and mitochondria; (2) the neuron and axon; (3) sodium channels; (4) microglia and astroglia; (5) intrathecal B cells and plasma cells; (6) demyelination and myelin repair; and (7) antioxidants. It was also agreed that the process of drug selection should be iterative, using a modified Delphi method, led by expert opinion within treatment selection group, while at each stage independent expert input would be sought.

We then convened an international treatment selection workshop, held in London in April 2018. Leading experts from the research community gave a series of talks in each mechanistic area and were asked to suggest drugs for consideration. We also invited representatives of the Cure Parkinson's Trust, the Alzheimer's Society, Motor Neuron Disease Association, Parkinson's UK and Medicines Discovery Catapult (MDC), who had undertaken drug repurposing programmes within their own disease area. ${ }^{14}{ }^{15}$ We agreed to draw up a template (a 'drug Curriculum Vitae (CV)') for each compound based on the Cure Parkinson's Trust linked clinical trials initiative dossier model. These documents included information on pharmacodynamics, pharmacokinetics, mechanism of action and evidence base in vitro, in vivo and in clinical trials (box 1). This CV condensed and systematised the literature on each drug into an accessible summary manuscript; a drug CV for each potential treatment would be completed by at least two members of the treatment selection group.

There was then a call for suggestions for repurposed drugs to members of the committee, clinicians, experts from the wider MS research community, people with MS and the public, via a web-based system that was advertised to the MS Society's mailing lists. Contributors were prompted to describe the scientific rationale for their proposed intervention. After 4 months, the call was closed, a long list of drugs was compiled, and drug $\mathrm{CV}$ s were completed for each.

The scientific members of the committee scored each drug CV according to an agreed system prioritising safety and efficacy (box 2). Members of the MS Society's RN also scored each drug for ease of administration, tolerability, safety and monitoring requirements based on the drug CV and the European Medicines Agency's (EMA) patient information leaflets (box 2). The scores were collated before a second face-to-face meeting.

At this meeting, in September 2018, the treatment selection group (voting) members were joined by new members of the research community and RN (invited attendees) to provide a fresh perspective on the drug list. Each drug was presented, discussed and given an overall score (between 0 as lowest and 5 as highest). The results were further reviewed and discussed, before all attendees ranked their top five drugs, which resulted in a list of seven prioritised drugs.

In parallel to the pilot stage of treatment selection, the MS Society commissioned MDC to independently identify licensed drugs that might impact progressive MS. This was undertaken to scrutinise our long list of drugs that had been compiled through 


\section{Box 1 Information recorded in the drug CV}

Summary information

- Drug name.

- Regulatory status.

- Mechanistic target.

- Dose for human use (and appropriateness for multiple sclerosis (MS)).

- Key safety concerns.

- Intellectual property.

- Outstanding critical issues.

- Overall evaluation.

Absorption, distribution, metabolism, excretion, pharmacokinetics and pharmacodynamics

- Chemical structure.

- Molecular target.

- Pathway affected.

- Human pharmacodynamics.

- Human pharmacokinetics.

- Blood-brain barrier penetrance.

- Route of administration.

- Licensed indication.

- Dose for licensed indication.

- Dose suitability for MS.

- Known, or anticipated, drug-drug interactions.

Scientific rationale

- Efficacy in in vitro models.

- Efficacy in in vivo models.

- Efficacy for primary indication.

- Efficacy in people with MS (if applicable).

- Particular subgroups of people with MS likely to benefit (if applicable).

Safety

- Animal safety issues.

- Therapeutic ratio (if known).

- Safety record in humans.

- Safety record in people with MS.

- Monitoring requirements.

- Any particular drug-drug interaction that would limit use in MS.

Landscape review

- Is there active preclinical research on the use of this drug in MS? Where?

- Has the Progressive MS Alliance prioritised this drug?

- Are there any relevant trials listed on clinical trials databases?

the aforementioned mechanism of drug proposals. MDC searched for all ongoing, or completed, trials in people with MS to identify drugs being tested for any type of MS. They then characterised their molecular targets and sought other compounds that were predicted to impact these targets. The final list was pruned of immunotherapies and symptomatic drugs, as well as those that did not cross the blood-brain barrier, and any not on the original long list were added for consideration during the final stage of treatment selection.

\section{Final stage of treatment selection}

The treatment selection group appointed new members and some original members left, leaving 13 scientific and 6 research network members. A renewed call for drug proposals was opened and the newly formed group reviewed any new

\section{Box 2 Scoring system for shortlisting drug CVs}

For scientific members of the panel

Safety: are the safety data for the treatment satisfactory? To include any regulatory warnings, adverse events, drug-drug interactions, therapeutic index and safety profile. (Score 0-2) Efficacy: do we have sufficient evidence that the treatment is likely to be effective in slowing progression? To include in vitro and in vivo experimental models, blood-brain barrier penetration, along with human data where available. (Score 0-2) Overall evaluation - priority level for the treatment (select one)

1. Licensed drug, ready for a phase II trial in MS, high priority.

2. Licensed drug, ready for a phase II trial in MS, low priority.

3. Licensed drug, with critical issues to be resolved before a phase II trial in multiple sclerosis (MS).

4. Interesting drug, with considerable preclinical work to be done.

5. Poor scientific rationale: not to be prioritised.

For research network members (people with MS)

Administration: is the method of taking the drug acceptable?

To consider whether it is a tablet, injection or infusion as well as how often it needs to be taken. (Score 0-2)

Side effects and risks: is the safety of the drug acceptable? To consider both the immediate side effects and risks as well as the long term. (Score 0-2)

Overall evaluation - priority level (select one)

1. I would take this drug even if it only moderately slowed the progression of my MS.

2. I would take this drug if it stopped the progression of my MS.

3. I would not take this drug even if it stopped the progression of my MS.

suggested compounds, the original long list of drugs considered during the pilot stage and those generated by MDC, resulting in a new long list of 29 drugs. Each of these had a drug CV compiled or updated by a team of four, two with a scientific background and two clinicians. The 13 scientific members of the treatment selection group then scored each drug CV according to a simplified scoring system based on safety, efficacy and an overall assessment of priority (box 2). Similarly, $6 \mathrm{RN}$ members of the treatment selection group and an additional 10 invited RN members scored between 5 and 10 of the drug CVs, with additional access to the EMA-approved patient information leaflet, such that at least five scores were recorded for each drug. The highest ranked 12 drugs from the collated scores formed the shortlist for a third face-to-face meeting in September 2019 of the treatment selection group, with a new group of invited experts and people with MS. Members of the treatment selection group had the option to rescue a low-scoring drug in advance of the meeting by presenting a case for its inclusion and it being accepted by majority vote. For the meeting, each drug was presented by one scientific and one research network member, who focused on the scientific case and attractiveness to people with MS, respectively. Drugs were then scored out of 5, and the resulting ranking discussed before each attendee individually ranked up to five drugs ready for use in a clinical trial.

The drug CVs of the treatments recommended by this meeting, and the two highest scoring drugs in the sodium channel antagonist class, were sent to four independent international MS experts outside the UK to achieve a further layer of scrutiny of the decisions and to elicit any information on the drugs that was not publicly available. Their comments were collated and 


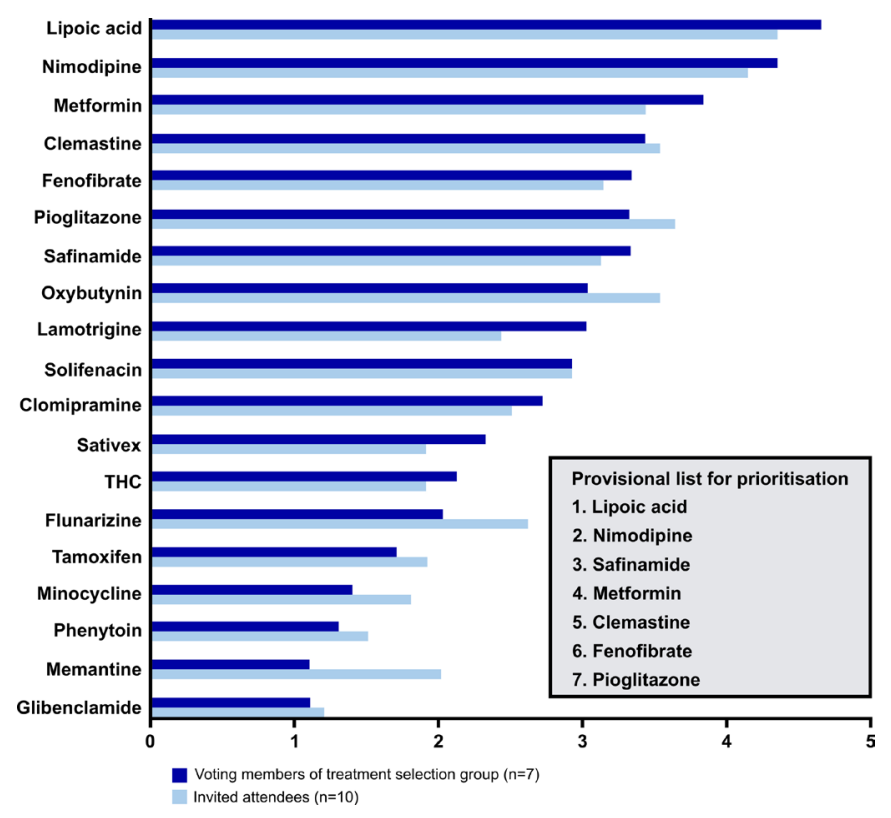

Figure 2 Outcome of the pilot screen of candidate interventions. Mean scores (out of five) of each drug by voting members of the treatment selection group and invited atendees are displayed in descending order. Inset: the provisional list for prioritisation agreed by the voting members of the committee.

considered alongside the outcome of the final treatment selection meeting by the Treatment Advisory Committee (figure 1). This committee advised on the final drug selection for the MS Society's Efficient Clinical Trials Platform, which is intended to evaluate repurposed treatments quickly and affordably. This committee comprised six scientific members and three people affected by MS. They assessed the prioritised list on the basis of scientific evidence and also in the context of other trials known to be going ahead elsewhere. They also scrutinised drug mechanisms and whether the chosen trial design and outcome measures would allow detection of treatment effects. This facilitated a final decision to be made for the drugs to be tested in a platform trial (figure 1).

\section{RESULTS}

\section{Pilot stage of treatment selection}

Forty-four treatments were proposed during the 2018 call for drug suggestions, with at least one believed to act on each of the target mechanisms. Thirty-five were deemed by the treatment selection group to have sufficient scientific rationale for consideration, and drug CVs were completed by its scientific members. Each was then scored, prioritising considerations of efficacy and safety as detailed in box 2, leading to a shortlist of 19 compounds to be discussed face to face in September 2018. At that meeting, each drug was presented and discussed before being scored again, collated separately for the members of the treatment selection group (voting members) and the invited attendees (experts and people affected by MS) (figure 2). After open discussion of these scores, the treatment selection group members ranked their preferred seven drugs.

During this pilot stage, we learnt that the drug CVs were effective but needed more consistency in authorship to promote comparable levels of detail in each $\mathrm{CV}$, and multiple contributors from different backgrounds to encourage impartiality in the presentation of the literature for each compound. We also reflected on the valuable contributions from people affected by
MS, who were in a unique position to weigh the safety and tolerability of each drug and consider the level of benefit they would require to take the proposed treatment for their MS. The group resolved that more research network members should be invited onto the treatment selection group to maximise representation of different viewpoints from within the MS community and to share the burden of scoring CVs and presenting drugs at meetings beyond the two original members.

\section{Final stage of treatment selection}

MDC identified 320 licensed drugs that had a mechanism similar to a drug that had been tried in MS. ${ }^{16}$ Once immunotherapies, drugs that did not cross the blood-brain barrier, and duplicates were removed, guanabenz and trazodone remained from this list. These were added to the 44 treatments that emerged from the pilot phase. During the renewed call for proposals in 2019, new members of the treatment selection committee and outside experts contributed these new suggestions: domperidone, benztropine, prednisolone, ibudilast, spironolactone, oxcarbazepine, hydroxychloroquine, niacin and the combination of metformin and R- $\alpha$-lipoic acid (R-ALA). This long list of 55 treatments was screened by the new treatment selection group, and 28 drugs and one combination therapy were chosen to have comprehensive drug CVs completed.

Twelve scientific members of the group scored all 29 drug CVs and $16 \mathrm{RN}$ members (six of which were members of the treatment selection group) scored up to 10 of the drug CVs, with additional access to the EMA-approved patient information leaflet, such that five RN scores were recorded for each drug. The scientific scores were ranked, and 13 drugs and 1 combination treatment (metformin and R-ALA) were shortlisted. If a scientific member disagreed with a drug excluded at this stage, they were able to make a case for its inclusion to the group and add to the shortlist by majority vote. Flunarizine and lamotrigine, which had initially been excluded from the list of 12 at the CV scoring stage, were readded to the list in this way.

The 14 shortlisted treatments were discussed and scored, one by one, at a face-to-face meeting of the treatment selection group and invited attendees. The collated scores (figure 3) were then discussed and debated before the treatment selection group ranked up to five drugs, which were ready for immediate use in a phase II clinical trial. The final shortlist list of drugs were, in order of preference: R-ALA, metformin, the combination treatment of R-ALA and metformin, and clemastine. We considered that niacin, flunarizine and nimodipine were particularly promising, but the treatment selection group felt they needed more preclinical work.

This selection, in addition to the two highest scoring sodium channel antagonist drugs (lamotrigine and oxcarbazepine), was sent to four independent expert reviewers. They scored each compound on safety and efficacy and ranked the drugs by priority level. They were also asked to provide information on any of these drugs that was not publicly accessible. The results of this procedure were considered by the Treatment Advisory Committee of the MS Society's Efficient Clinical Trials Platform (figure 1), and a final order of prioritisation was made (table 1). The top four were recommended as the most promising for clinical evaluation. The pathway of each drug through these procedures is summarised in figure 4 .

\section{DISCUSSION}

The pathogenesis of progressive MS is complex, multifaceted and poorly understood. As with many other neurodegenerative 


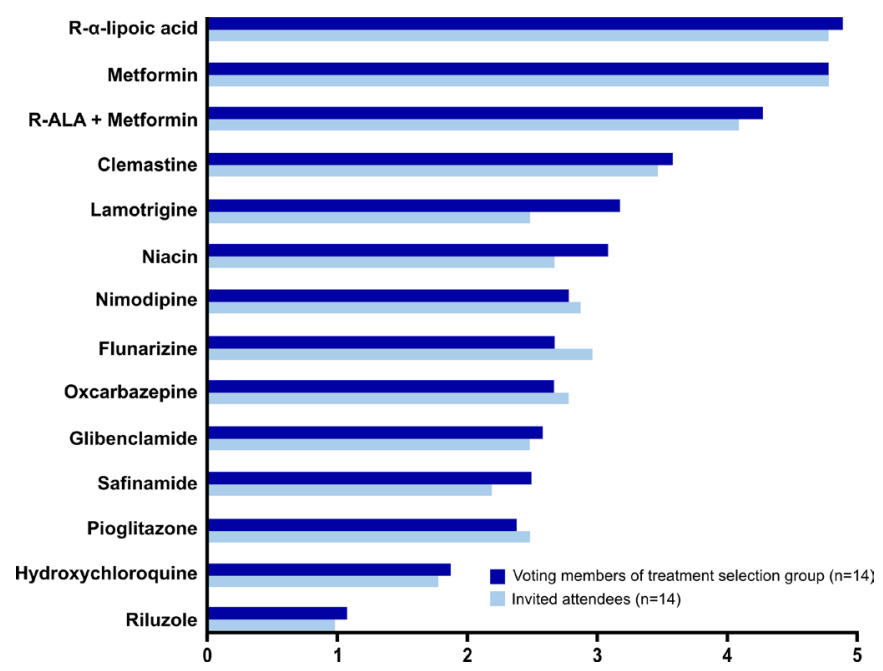

Figure 3 Outcome of the final meeting of the treatment selection group during the final stage of candidate screening. The mean scores (out of 5) for the 14 shortlisted compounds presented at the meeting are divided into those awarded by voting members of the panel and invited attendees.

diseases, there are no licensed treatments. This remains the greatest unmet need for the more than 2.3 million people affected by MS globally. ${ }^{17}$ Placed in context of the high cost, long time and high attrition rate from target selection to regulatory approval via conventional pathways, there are compelling reasons to explore opportunities provided by drug repurposing. This nevertheless presents a substantial challenge. The myriad reasons for the prior failure to find an effective treatment remain, ${ }^{6}$ and the optimum process for selection of drugs to progress to repurposing clinical trials are not standardised. Procedures for synthesising experimental and clinical trial data

\begin{tabular}{|c|c|}
\hline $\begin{array}{l}\text { Final list of drugs for } \\
\text { prioritisation }\end{array}$ & Mechanism of action \\
\hline 1. R- $\alpha$-lipoic acid* & $\begin{array}{l}\text { Dietary supplement, approved in Germany for diabetic } \\
\text { neuropathy; antioxidant, anti-inflammatory and } \\
\text { neuroprotective. }\end{array}$ \\
\hline 2. Metformint & $\begin{array}{l}\text { Antihyperglycaemic agent used for type } 2 \text { diabetes } \\
\text { mellitus; anti-inflammatory }{ }^{23} \text { and promotes } \\
\text { remyelination }^{25} \text { and neuroprotection. }{ }^{24}\end{array}$ \\
\hline $\begin{array}{l}\text { 3. R- } \alpha \text {-lipoic acid and } \\
\text { metformin combination }\end{array}$ & $\begin{array}{l}\text { Mechanisms as above; complimentary mechanistic targets } \\
\text { and neuroprotective in combination. }\end{array}$ \\
\hline 4. Niacinł & $\begin{array}{l}\text { Antihypercholesterolaemic drug; promotes } \\
\text { oligodendrocyte proliferation, }{ }^{29} \text { remyelination }{ }^{30} \text { and } \\
\text { neuroprotection. }{ }^{28}\end{array}$ \\
\hline 5. Clemastine & $\begin{array}{l}\text { Antihistamine used for allergic rhinitis; off-target anti- } \\
\text { muscarinic (M1) action, which promotes oligodendrocyte } \\
\text { progenitor differentiation and remyelination. }{ }^{32} 33\end{array}$ \\
\hline 6. Lamotrigine & $\begin{array}{l}\text { Sodium channel antagonist widely used as an } \\
\text { anticonvulsant; neuroprotective effects. }{ }^{36}\end{array}$ \\
\hline 7. Oxcarbazepine & $\begin{array}{l}\text { Sodium channel antagonist widely used as an } \\
\text { anticonvulsant; neuroprotective effects. }{ }^{37}\end{array}$ \\
\hline 8. Nimodipine & $\begin{array}{l}\text { Calcium channel antagonist used to treat vasospasm in } \\
\text { subarachnoid haemorrhage; promotes remyelination, } \\
\text { neuroprotection }{ }^{38} \text { and restores CNS perfusion and } \\
\text { oxygenation. }{ }^{39}\end{array}$ \\
\hline 9. Flunarizine & Migraine prophylactic; neuroprotective effects. ${ }^{40}$ \\
\hline \multicolumn{2}{|c|}{$\begin{array}{l}\text { The top four were determined to be the most promising for clinical evaluation. } \\
\text { * } 1200 \mathrm{mg} / \text { day. } \\
\$ 1 \mathrm{~g} \text { twice daily, starting at } 500 \mathrm{mg} \text { twice daily. } \\
\$ 750 \mathrm{mg} \text { twice daily of slow release formulation of Niaspan. } \\
\text { CNS, central nervous system. }\end{array}$} \\
\hline
\end{tabular}

to enable rational drug selection are required to maximise the chance of successful clinical development.

The UK MS Society Clinical Trials Network was initiated in 2007 and commissioned key underpinning work including a review of animal and human data on promising drugs. Given the mechanistic overlap between SPMS and other neurodegenerative disorders (namely Alzheimer's disease, Parkinson's disease, Huntington's disease and amyotrophic lateral sclerosis), their strategy centred on a systematic review and meta-analysis of clinical and preclinical data for agents previously tested in these illnesses..$^{9}$ The ensuing list prioritised ibudilast, riluzole, amiloride, fluoxetine, pirfenidone, oxcarbazepine and agents of the PUFA class. Ibudilast and lipoic acid proved successful at phase II, ${ }^{1011}$ but unfortunately, riluzole, amiloride and fluoxetine did not reduce brain atrophy in the MS-SMART study compared with placebo. ${ }^{1213}$

Here we describe a rigorous, expert-led, evidence-based approach to the selection of licensed compounds for repurposing in clinical trials of people with progressive forms of MS, led by scientific and clinical experts as well as people with MS, involving repeated rounds of assessment, scoring and independent peer review. We identified key biological mechanisms, performed an exhaustive literature search on identified drugs and went through two cycles of shortlisting and prioritisation. We selected this strategy to retain the evidence-based approach of previous mechanisms of drug selection, but with added emphasis on expert opinion and independent expert review which, in our view, would enable our selection to be based on current scientific opinion and more readily identify barriers and knowledge gaps that might affect trials of the proposed compounds. A particular contrast between our strategy and that previously used was that we did not prioritise agents that had previously been subject of clinical trials of people with neurodegenerative illnesses, and we required all candidates to have evidence of blood-brain barrier permeability. Other differences are summarised in table 2.

It is noteworthy that our first ranked drug, lipoic acid, was also prioritised in the 2011 drug selection initiative, despite the contrasting methodologies. Three interventions-R-ALA, metformin and niacin-and one combination preparation-of metformin and R-ALA-were identified as being priorities for clinical evaluation in cohorts of people with progressive MS and as having sufficient data to permit immediate entry into a phase II trial. Clemastine, lamotrigine, oxcarbazepine, nimodipine and flunarizine were also felt to be promising and ranked in order of priority.

R-ALA is the R-enantiomer that makes up 50\% of the racemic mixture ( $\mathrm{R}$ and $\mathrm{S}$ ) of lipoic acid, a dietary supplement approved in Germany for the treatment of diabetic neuropathies. It has previously been shown to be a potent antioxidant, have antiinflammatory properties, ${ }^{18}{ }^{19}$ and reduce excitotoxic damage, ${ }^{20}$ while the $\mathrm{R}$ enantiomer has superior pharmacokinetic, antioxidant and neuroprotective properties than the $S$ enantiomer. ${ }^{21}$ When given to 51 people with SPMS, it was shown to have a small benefit to brain atrophy. ${ }^{22}$

Metformin, a biguanide licensed for human use in type 2 diabetes, has previously been demonstrated to reduce inflammation in progressive and relapsing experimental autoimmune encephalomyelitis models, ${ }^{23}$ is neuroprotective in models of glucose deprivation/reoxygenation ${ }^{24}$ and, more recently, has been shown to reverse an age-associated barrier to the ability of oligodendrocyte progenitor cells to respond to differentiation factors and facilitate subsequent remyelination. ${ }^{25}$ Additionally, it has previously been used in 20 people with MS and demonstrated a reduction in the number of new or enlarging T2 lesions 

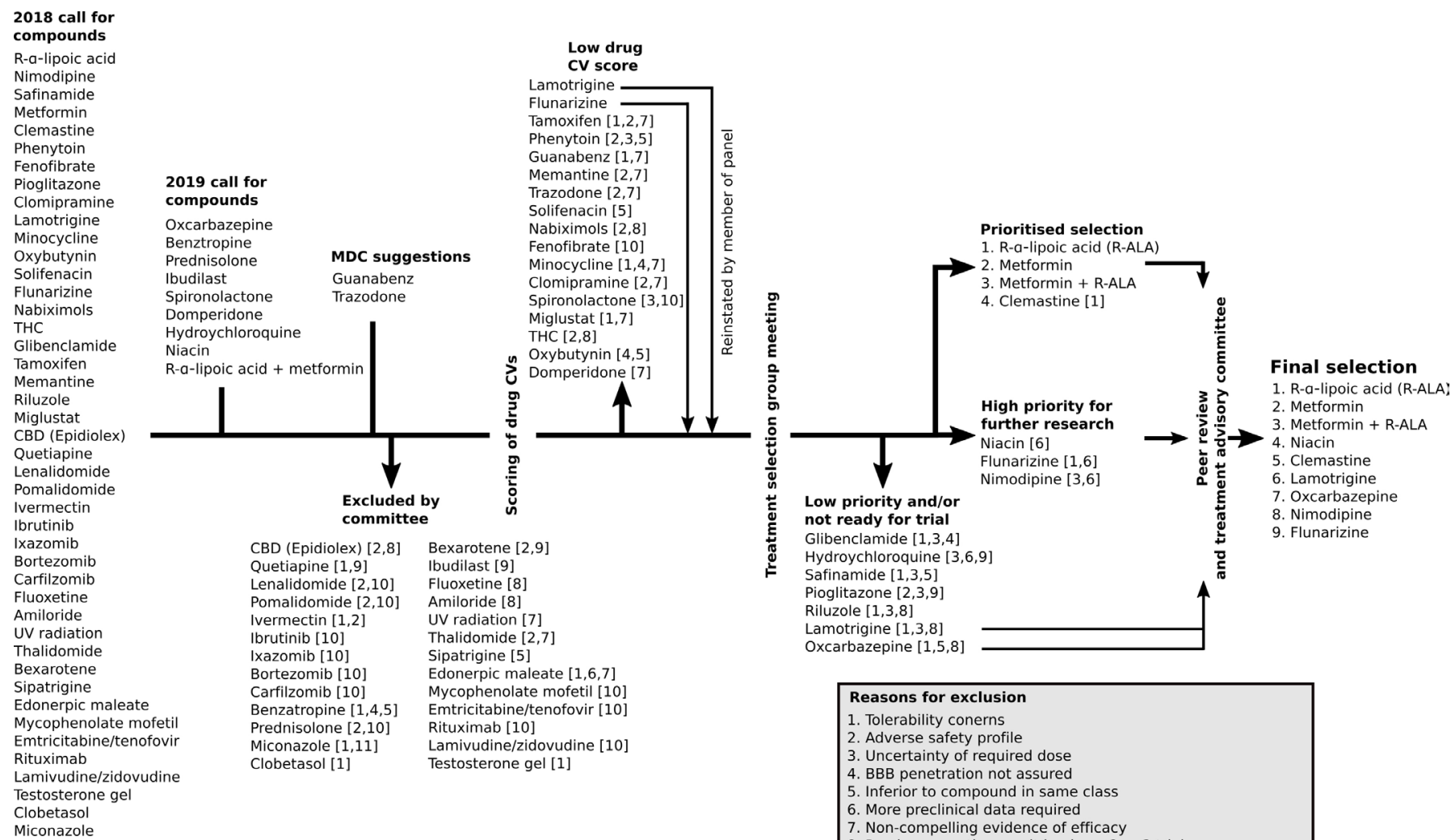

\begin{tabular}{|l|}
\hline Reasons for exclusion \\
1. Tolerability conerns \\
2. Adverse safety profile \\
3. Uncertainty of required dose \\
4. BBB penetration not assured \\
5. Inferior to compound in same class \\
6. More preclinical data required \\
7. Non-compelling evidence of efficacy \\
8. Previous negative result in phase 2 or 3 trial \\
9. Current subject of clinical trial \\
10. Limited plausibility outside immunosuppressive mechanism \\
11. Lack of oral preparation
\end{tabular}

Figure 4 Summary of the pathway of each drug through the treatment selection process to yield a final prioritised list of drugs. BBB, blood-brain barrier.

compared with placebo. $^{26}$ The complimentary mechanistic targets of metformin and R-ALA, as well as the potential for synergy, ${ }^{27}$ led to the combination of the two featuring on our prioritised list.

Niacin, a nicotinamide adenine dinucleotide precursor in use for the treatment of hypercholesterolaemia, has previously been shown to be protective against activated microglial-induced neurotoxicity ${ }^{28}$ and to promote oligodendrocyte proliferation in vitro. ${ }^{29}$ In line with these observations, it reduces axonal degeneration, delays progression, and increases oligodendrocyte proliferation in extrinsic allergic encephalomyelitis. ${ }^{28}{ }^{29}$ While ranked below clemastine by the treatment selection group, data that was unpublished at the time came to light during the treatment advisory committee review: niacin also enhances myelin

Table 2 Comparison between the current methodology and that previously used in $2011^{9}$

\begin{tabular}{|c|c|c|}
\hline & 2011 & 2019 \\
\hline Method of drug identification & $\begin{array}{l}\text { Thorough and systematic search of online databases (PubMed, ISI Web } \\
\text { of Knowledge, Embase, Clinicaltrials.gov and Cochrane MS group). }\end{array}$ & $\begin{array}{l}\text { Calls for recommendations from academics, clinicians and people with MS. } \\
\text { Systematic search of online databases by medicines discovery catapult. }\end{array}$ \\
\hline Previous clinical trial use & $\begin{array}{l}\text { Previously used in a neurodegenerative disease including progressive } \\
M S, P D, H D, A D \text {, and } A L S \text {. }\end{array}$ & Human safety data required only. \\
\hline Mechanistic targets & $\begin{array}{l}\text { Excluded immunosuppressant mechanism of action. } \\
\text { Combination treatments excluded. }\end{array}$ & $\begin{array}{l}\text { Priority given to candidates targeting several mechanistic targets. } \\
\text { Excluded those with solely immunosuppressant mechanism. } \\
\text { Combination treatments accepted. }\end{array}$ \\
\hline Method of administration & Oral. & Any method of administration. \\
\hline CNS penetration & Reviewed at selection meeting. & Evidence of BBB permeability required at study entry. \\
\hline Safety & $\begin{array}{l}\text { Excluded those with significant adverse effects associated with } \\
\text { treatment. }\end{array}$ & Excluded those with significant adverse effects associated with treatment. \\
\hline Method of selection & $\begin{array}{l}\text { Systematic evaluation of publications pertaining to each candidate. } \\
\text { Systematic review of experimental autoimmune encephalomyelitis } \\
\text { preclinical data for each candidate. } \\
\text { Scrutiny of each drug by an international multidisciplinary committee. }\end{array}$ & $\begin{array}{l}\text { Systematic evaluation of preclinical and clinical publications pertaining to } \\
\text { each candidate. } \\
\text { Formation of a database of drug CVs. } \\
\text { Rating of these by scientific panel. } \\
\text { Presentation and decision at international multidisciplinary meeting. }\end{array}$ \\
\hline Input from people affected by MS & Patient representatives acting as external advisors. & $\begin{array}{l}6 \text { members of MS research network on voting panel. } \\
\text { Scoring of drug CVs by at least five people with, or affected by, MS. } \\
\text { Members of MS research network at treatment selection meeting. }\end{array}$ \\
\hline Peer review & $\begin{array}{l}\text { External advisors with a range of expertise including animal models, } \\
\text { disease biology, clinical trial design, systematic review and patient } \\
\text { representation. }\end{array}$ & Methodology and final treatment selection sent for external peer review. \\
\hline
\end{tabular}

AD, Alzheimer's disease; ALS, amyotrophic-lateral sclerosis; BBB, blood-brain barrier; CNS, central nervous system; HD, Huntington's disease; MS, multiple sclerosis; PD, Parkinson's disease. 
phagocytosis by microglia, leading to increases in oligodendrocyte progenitor cell numbers and improved remyelination in mice. ${ }^{30}$ Niacin has not yet been trialled in people with MS.

Finally, clemastine is a first-generation antihistamine that was identified in two separate screens as being able to stimulate oligodendrocyte progenitor cells to differentiate and carry out the first stages of remyelination. ${ }^{31} 32$ This subsequently demonstrated a small, but statistically significant, improvement in the latency of the full-field visual evoked potential of people with relapsing MS and chronic stable optic neuropathy, interpreted as a remyelinating effect in the optical pathway. ${ }^{33}$

A particular strength of our methodology is the multiple layers of revision and review. By undertaking a pilot of treatment selection, we refined the procedures by which we evaluated the literature and assessed each compound to facilitate robust comparisons of agents with disparate mechanistic targets and safety profiles. We also ratified our procedures for drug identification by the work of MDC, which generated a list of drugs of which only two had not previously been identified. Finally, by sending our list of prioritised treatments for external peer review, we have better ensured scrutiny of both our methods and our selection.

\author{
Author affiliations \\ 'Department of Clinical Neurosciences, University of Cambridge, Cambridge, UK \\ ${ }^{2}$ Multiple Sclerosis Society, London, UK \\ ${ }^{3}$ Research Network, Multiple Sclerosis Society, London, UK \\ ${ }^{4}$ Blizard Institute, Queen Mary University of London, London, UK \\ Independent consultant, Stevenage, UK \\ ${ }^{6}$ Department of Neurology, University of Oxford, Oxford, UK \\ 'Wellcome-Wolfson Institute for Experimental Medicine, Queen's Univeristy, Belfast, \\ ${ }^{8}$ Faculty of Brain Sciences, Queen Square Institute of Neurology, University College \\ London, London, UK \\ ${ }^{9}$ Centre for Clinical Brain Sciences, Anne Rowling Regenerative Neurology Clinic, \\ University of Edinburgh, Edinburgh, UK \\ ${ }^{10}$ University of Reading, Reading, UK \\ ${ }^{11}$ Independent consultant, Woodstock, Oxfordshire, UK \\ ${ }^{12}$ Department of Neuroinflammation, Queen Square Institute of Neurology, University \\ College London, London, UK \\ ${ }^{13}$ Department of Neurology, University of Bristol, Bristol, UK \\ ${ }^{14} \mathrm{MS}$ Centre, Centre for regenerative medicine, University of Edinburgh, Edinburgh,
}

\section{Twitter Nick Cunniffe @NickGCunniffe}

Contributors NC and AC accept full responsibility for the work and conduct of the study, had access to the data and controlled the decision to publish. Study concept and design: all authors. Members of treatment selection group responsible for analysis or interpretation of data: all authors. Drafting of manuscript: NC, KAV and AC. Critical revision of the manuscript: all authors.

Funding JB received expense payments from Novartis for speaking as patient representative during Siponimod licensing. AC receives funding from the Medical Research Council (MRC) and MS Society UK. DF is funded by the Biotechnology and Biological Sciences Research Council (BBSRC) and has a project with Sangamo. AGdlF has been supported by the European Committee for Treatment and Research in MS (ECTRIMS) postdoctoral fellowship during this period. GG declares current research funding from Merck KGa (CLAD-B study), Roche (ORATORIO-HAND study) and Takeda (SIZOMUS Study). DM received funding previously from Biogen, MedDay and SanofiGenzyme. BN received funding from the Cambridge Centre for Myelin Repair, funded by MS Society UK. SP declares current funding from Italian and US Multiple Sclerosis Societies. LP-J has been supported by a senior research fellowship FISM —Fondazione Italiana Sclerosi Multipla — cod. 2017/B/5 and financed or co financed with the '5five per mille' public funding, by the Isaac Newton Trust RG 97440 and the Addenbrooke's Charitable Trust RG 97519. KJS declares current funding from Fondation Leducq, Multiple Sclerosis Society, Rosetrees Trust. AW received a research grant from Sanofy (2018). AW declares funding from MS Society UK, Roche, MRC and Lifearc.

Competing interests DB received compensation for consultancy activity from Canbex Therapeutics, Japan Tobacco, Lundbeck, InMune Bio, Merck, Novartis, and Roche in the past 3 years. AC received honoraria and travel support from Genzyme (a Sanofi company) prior to 2017. MC has received honoraria for educational events and/or consultancy from Biogen, Merck, Roche, AbbVie and Novartis. GG has received compensation for serving as a consultant in relation to multiple sclerosis drug development from AbbVie, Actelion, Atara Bio, Biogen, Celgene, EMD Serono, Japanese Tobacco, Sanofi-Genzyme, Genentech, GlaxoSmithKline, GW Pharma, Merck KGa, Novartis, Roche and Teva. LH holds a small number of GSK shares as part of her renumeration when she was an employee, which she left 4 years ago. DM received consultancy fees from Biogen, MedDay and SanofiGenzyme and Novartis. BN holds a patent regarding the treatment of demyelinating diseases including metformin: W02019/206419 A1, Treatment for demyelinating disease. SP is cofounder, CSO and shareholder ( $>5 \%$ ) of CITC Ltd and iSTEM Therapeutics, and cofounder and non-executive director at Asitia Therapeutics. LP-J is Head of Research at iSTEM Therapeutics. AW receives research support from Roche not associated with drug development or use.

Patient consent for publication Not required.

Provenance and peer review Not commissioned; externally peer reviewed.

Data availability statement Data are available on reasonable request. All authors had access to the data in this study and had responsibility for the decision to submit for publication. All data relevant to the study are included in the article or uploaded as supplementary information. Information recorded in the drug CVs is available from the MS Society and can be requested via email: research@mssociety. org.uk.

\section{ORCID iDs}

Nick Cunniffe http://orcid.org/0000-0002-7562-2838

David Baker http://orcid.org/0000-0002-8872-8711

Gavin Giovannoni http://orcid.org/0000-0001-9995-1700

\section{REFERENCES}

1 Compston A, Coles A. Multiple sclerosis. Lancet 2008;372:1502-17.

2 Scolding N, Barnes D, Cader S, et al. Association of British neurologists: revised (2015) guidelines for prescribing disease-modifying treatments in multiple sclerosis. Pract Neuro/ 2015;15:273-9.

3 Weinshenker BG, Bass B, Rice GP, et al. The natural history of multiple sclerosis: a geographically based study. I. clinical course and disability. Brain 1989;112:133-46

4 Montalban X, Hauser SL, Kappos L, et al. Ocrelizumab versus placebo in primary progressive multiple sclerosis. N Engl J Med 2017;376:209-20.

5 Kappos L, Bar-Or A, Cree BAC, et al. Siponimod versus placebo in secondary progressive multiple sclerosis (expand): a double-blind, randomised, phase 3 study. Lancet 2018;391:1263-73.

6 Faissner S, Plemel JR, Gold R, et al. Progressive multiple sclerosis: from pathophysiology to therapeutic strategies. Nat Rev Drug Discov 2019;18:905-22.

7 O'Connor KA, Roth BL. Finding new tricks for old drugs: an efficient route for publicsector drug discovery. Nat Rev Drug Discov 2005;4:1005-14.

8 Pushpakom S, lorio F, Eyers PA, et al. Drug repurposing: progress, challenges and recommendations. Nat Rev Drug Discov 2019;18:41-58.

9 Vesterinen HM, Connick P, Irvine CMJ, et al. Drug repurposing: a systematic approach to evaluate candidate oral neuroprotective interventions for secondary progressive multiple sclerosis. PLoS One 2015;10:e0117705.

10 Fox RJ, Coffey CS, Conwit R, et al. Phase 2 trial of ibudilast in progressive multiple sclerosis. N Engl J Med 2018;379:846-55

11 Spain R, Powers K, Murchison C, et al. Lipoic acid in secondary progressive MS. Neurol Neuroimmunol Neuroinflamm 2017;4:e374.

12 Connick P, De Angelis F, Parker RA, et al. Multiple sclerosis-secondary progressive multi-arm randomisation trial (MS-SMART): a multiarm phase IIb randomised, doubleblind, placebo-controlled clinical trial comparing the efficacy of three neuroprotective drugs in secondary progressive multiple sclerosis. BMJ Open 2018;8:e021944.

13 Chataway J, De Angelis F, Connick $\mathrm{P}$, et al. Efficacy of three neuroprotective drugs in secondary progressive multiple sclerosis (MS-SMART): a phase $2 \mathrm{~B}$, multiarm, doubleblind, randomised placebo-controlled trial. Lancet Neurol 2020;19:214-25.

14 Brundin P, Barker RA, Conn PJ, et al. Linked clinical trials--the development of new clinical learning studies in Parkinson's disease using screening of multiple prospective new treatments. J Parkinsons Dis 2013;3:231-9.

15 Corbett A, Pickett J, Burns A, et al. Drug repositioning for Alzheimer's disease. Nat Rev Drug Discov 2012;11:833-46.

16 Medicines Discovery Catapult. Using a data-driven approach to identify drug candidates for multiple sclerosis. Available: https://md.catapult.org.uk/case-studies/ using-a-data-driven-approach-to-identify-drug-candidates-for-multiple-sclerosis/ [Accessed 27 Aug 2020].

17 Browne P, Chandraratna D, Angood C, et al. Atlas of multiple sclerosis 2013: a growing global problem with widespread inequity. Neurology 2014;83:1022-4.

18 Cho YS, Lee J, Lee T-H, et al. Alpha-lipoic acid inhibits airway inflammation and hyperresponsiveness in a mouse model of asthma. J Allergy Clin Immunol 2004;114:429-35.

19 Liu W, Shi L-J, Li S-G. The immunomodulatory effect of alpha-lipoic acid in autoimmune diseases. Biomed Res Int 2019;2019:1-11. 
20 Park E, Gim J, Kim DK, et al. Protective effects of alpha-lipoic acid on glutamateinduced cytotoxicity in C6 glioma cells. Biol Pharm Bull 2019;42:94-102.

21 Tomassoni D, Amenta F, Amantini C, et al. Brain activity of thioctic acid enantiomers: in vitro and in vivo studies in an animal model of cerebrovascular injury. Int J Mol Sci 2013;14:4580-95.

22 Loy BD, Fling BW, Horak FB, et al. Effects of lipoic acid on walking performance, gait, and balance in secondary progressive multiple sclerosis. Complement Ther Med 2018:41:169-74.

23 Nath N, Khan M, Paintlia MK, et al. Metformin attenuated the autoimmune disease of the central nervous system in animal models of multiple sclerosis. J Immunol 2009;182:8005-14.

24 Meng X, Chu G, Yang Z, et al. Metformin protects neurons against oxygen-glucose deprivation/reoxygenation -induced injury by down-regulating MAD2B. Cell Physiol Biochem 2016;40:477-85.

25 Neumann B, Baror R, Zhao C, et al. Metformin restores CNS remyelination capacity by Rejuvenating aged stem cells. Cell Stem Cell 2019;25:473-85.

26 Negrotto L, Farez MF, Correale J. Immunologic effects of metformin and pioglitazone treatment on metabolic syndrome and multiple sclerosis. JAMA Neurol 2016:73:520-8

27 Ahuja S, Uniyal A, Akhtar A, et al. Alpha lipoic acid and metformin alleviates experimentally induced insulin resistance and cognitive deficit by modulation of TLR2 signalling. Pharmacol Rep 2019;71:614-23.

28 Kaneko S, Wang J, Kaneko M, et al. Protecting axonal degeneration by increasing nicotinamide adenine dinucleotide levels in experimental autoimmune encephalomyelitis models. J Neurosci 2006;26:9794-804.

29 Zhang J, Chen J, Li Y, et al. Niaspan treatment improves neurological functional recovery in experimental autoimmune encephalomyelitis mice. Neurobiol Dis 2008:32:273-80.
30 Rawji KS, Young AMH, Ghosh T, et al. Niacin-mediated rejuvenation of macrophage/ microglia enhances remyelination of the aging central nervous system. Acta Neuropathol 2020;139:893-909.

31 Deshmukh VA, Tardif $\mathrm{V}$, Lyssiotis $\mathrm{CA}$, et al. A regenerative approach to the treatment of multiple sclerosis. Nature 2013;502:327-32.

32 Mei F, Fancy SPJ, Shen Y-AA, et al. Micropillar arrays as a high-throughput screening platform for therapeutics in multiple sclerosis. Nat Med 2014;20:954-60.

33 Green AJ, Gelfand JM, Cree BA, et al. Clemastine fumarate as a remyelinating therapy for multiple sclerosis (rebuild): a randomised, controlled, double-blind, crossover trial. Lancet 2017;390:2481-9.

34 Marracci GH, Jones RE, McKeon GP, et al. Alpha lipoic acid inhibits T cell migration into the spinal cord and suppresses and treats experimental autoimmune encephalomyelitis. J Neuroimmunol 2002;131:104-14.

35 Morini M, Roccatagliata L, Dell'Eva R, et al. Alpha-lipoic acid is effective in prevention and treatment of experimental autoimmune encephalomyelitis. J Neuroimmunol 2004;148:146-53.

36 Bechtold DA, Miller SJ, Dawson AC, et al. Axonal protection achieved in a model of multiple sclerosis using lamotrigine. J Neurol 2006;253:1542-51.

37 Al-Izki S, Pryce G, Hankey DJR, et al. Lesional-targeting of neuroprotection to the inflammatory penumbra in experimental multiple sclerosis. Brain 2014:137:92-108

38 Schampel A, Volovitch 0 , Koeniger $\mathrm{T}$, et al. Nimodipine fosters remyelination in a mouse model of multiple sclerosis and induces microglia-specific apoptosis. Proc Natl Acad Sci U S A 2017:114:E3295-304.

39 Desai RA, Davies AL, Del Rossi N, et al. Nimodipine reduces dysfunction and demyelination in models of multiple sclerosis. Ann Neurol 2020;88:123-36.

40 Bostanci MO, Bağirici F, Canan S. A calcium channel blocker flunarizine attenuates the neurotoxic effects of iron. Cell Biol Toxicol 2006:22:119-25. 